\title{
UNSYMMETRICAL APPROXIMATION OF IRRATIONAL NUMBERS
}

\author{
RAPHAEL M. ROBINSON
}

1. Introduction. In a recent paper, B. Segre ${ }^{1}$ showed that for any $\tau \geqq 0$ an irrational number $\xi$ can be approximated by infinitely many fractions $A / B$ in such a way that

$$
-\frac{1}{(1+4 \tau)^{1 / 2} B^{2}}<\frac{A}{B}-\xi<\frac{\tau}{(1+4 \tau)^{1 / 2} B^{2}} .
$$

For $\tau=0$, this places $A / B$ to the left of $\xi$ and within a distance $1 / B^{2}$ from it. This type of approximation was known to be possible, since alternate convergents to the continued fraction representing $\xi$ satisfy this condition. For $\tau=1$, the inequality becomes

$$
-\frac{1}{5^{1 / 2} B^{2}}<\frac{A}{B}-\xi<\frac{1}{5^{1 / 2} B^{2}},
$$

so that we have the classical theorem of Hurwitz. ${ }^{2}$ For other values of $\tau$, approximations from both sides are permitted, but the errors allowed on the two sides are different; hence the term unsymmetrical approximation. The result here was new, and is so related to Hurwitz's inequality that one side is strengthened and the other weakened.

Notice that the result for $\tau>1$ is weaker than the result for $\tau<1$. For suppose that $\tau>1$, and apply the theorem with $\tau$ replaced by $1 / \tau$ to the irrational number $-\xi$. In this way, the permissible errors on the right and left are interchanged, and we see that $\xi$ has infinitely many approximations $A / B$ satisfying

$$
-\frac{1}{\left(\tau^{2}+4 \tau\right)^{1 / 2} B^{2}}<\frac{A}{B}-\xi<\frac{\tau}{\left(\tau^{2}+4 \tau\right)^{1 / 2} B^{2}},
$$

which is stronger than the original inequality. It is therefore sufficient to prove Segre's theorem for $0 \leqq \tau \leqq 1$.

Segre's proof depends on considering whether certain regions con-

\footnotetext{
Presented to the Society, November 2, 1946; received by the editors, October 8 , 1946.

${ }^{1}$ B. Segre, Lattice points in infinite domains, and asymmetric Diophantine approximations, Duke Math. J. vol. 12 (1945) pp. 337-365.

2 A. Hurwitz, Über die angenäherte Darstellung der Irrationalzahlen durch rationale Briiche, Math. Ann. vol. 39 (1891) pp. 279-284.
} 
tain lattice points. C. D. Olds ${ }^{3}$ has given a proof for the case $\tau \geqq 1$, making use of Farey series. We shall consider the problem, making use of continued fractions, and shall prove Segre's theorem (in \$6) and somewhat more. In particular, we shall show (in $\$ 7$ ) that for any $\epsilon>0$ the inequality

$$
-\frac{1}{\left(5^{1 / 2}-\epsilon\right) B^{2}}<\frac{A}{B}-\xi<\frac{1}{\left(5^{1 / 2}+1\right) B^{2}}
$$

has infinitely many solutions. This result is interesting since it shows that one side of Hurwitz's inequality can be strengthened without essentially weakening the other.

In $\S 2$ we state a few known results about continued fractions which we shall need to use, and in $\S \S 3-5$ we develop the theory that is needed for the last two sections. It may be remarked that $\$ 3$ is of some interest in itself, since we show there that the best approximations to an irrational number $\xi$ from either side are convergents to $\xi$.

2. Known results about continued fractions. Besides the convergents $A_{n} / B_{n}=\left[q_{0}, q_{1}, \cdots, q_{n}\right]$ to the continued fraction $\xi=\left[q_{0}, q_{1}\right.$, $\left.q_{2}, \ldots\right]$, we shall consider as approximations to $\xi$ the secondary convergents

$$
\begin{aligned}
& \frac{A_{n}+A_{n-1}}{B_{n}+B_{n-1}}=\left[q_{0}, \cdots, q_{n-1}, q_{n}+1\right], \\
& \frac{A_{n}-A_{n-1}}{B_{n}-B_{n-1}}=\left[q_{0}, \cdots, q_{n-1}, q_{n}-1\right] .
\end{aligned}
$$

However, in case $q_{n+1}=1$ the first reduces to the convergent $A_{n+1} / B_{n+1}$, and in case $q_{n}=1$ the second reduces to the convergent $A_{n-2} / B_{n-2}$; in such cases they will not be called secondary convergents. Notice that the first is on the opposite side of $\xi$ from $A_{n} / B_{n}$, while the second is on the same side as $A_{n} / B_{n}$.

If in general we put

$$
\left|\frac{A}{B}-\xi\right|=\frac{1}{\lambda B^{2}}
$$

then it is seen that for the convergent $A_{n} / B_{n}$ the value of $\lambda$ is

$$
\lambda_{n}=\beta_{n}+\frac{1}{\alpha_{n}},
$$

${ }^{3} \mathrm{C} . \mathrm{D}$. Olds, Note on an asymmetric Diophantine approximation, Bull. Amer. Math. Soc. vol. 52 (1946) pp. 261-263. 
and that for the secondary convergents considered, the values of $\lambda$ are

$$
\lambda_{n}^{\prime}=1+\frac{1}{\beta_{n}-1}-\frac{1}{\alpha_{n}+1}, \quad \lambda_{n}^{\prime}=1+\frac{1}{\alpha_{n}-1}-\frac{1}{\beta_{n}+1},
$$

respectively, where

$$
\alpha_{n}=\left[q_{n}, q_{n-1}, \cdots, q_{1}\right], \quad \beta_{n}=\left[q_{n+1}, q_{n+2}, \cdots\right] .
$$

We shall use the notation

$$
\left[\cdots, q_{-2}, q_{-1},\left[q_{0}\right], q_{1}, q_{2}, \cdots\right]
$$

to denote the sum of two continued fractions

$$
\left[q_{0}, q_{1}, q_{2}, \cdots\right]+\left[0, q_{-1}, q_{-2}, \cdots\right] .
$$

With this notation,

$$
\begin{aligned}
\lambda_{n} & =\left[q_{1}, \cdots, q_{n-1}, q_{n},\left[q_{n+1}\right], q_{n+2}, \cdots\right], \\
\lambda_{n}^{\prime} & =\left[q_{1}, \cdots, q_{n-1}, q_{n}, 1,[0], q_{n+1}-1, q_{n+2}, \cdots\right], \\
\lambda_{n}^{\prime \prime} & =\left[q_{1}, \cdots, q_{n-1}, q_{n}-1,[0], 1, q_{n+1}, q_{n+2}, \cdots\right] .
\end{aligned}
$$

Besides the classical results that every convergent represents $\xi$ with an error less than $1 / B^{2}$, and that every approximation for which the error is less than $1 / 2 B^{2}$ is a convergent, we shall also need the result that every approximation with an error less than $1 / B^{2}$ is either a convergent or a secondary convergent. ${ }^{4}$

3. The best approximations are convergents. A classical result about continued fractions is that the best approximations to an irrational number $\xi$ are included among the convergents to the continued fraction representing $\xi$. This can be understood in the following sense: The inequality

$$
\left|\frac{A}{B}-\xi\right|<\frac{1}{\mu B^{2}}
$$

has infinitely many solutions, all of which are convergents to $\xi$, if $\mu$ is a suitable number; indeed, $\mu$ may be any constant such that $2 \leqq \mu \leqq 5^{1 / 2}$.

We shall show that in a somewhat similar sense the best approximations to $\xi$ from the right are included among the convergents to $\xi$. More precisely, the inequality

4 This result was stated without proof by P. Fatou, Sur l'approximation des incommensurables et les séries trigonometriques, C. R. Acad. Sci. Paris vol. 139 (1904) pp. 1019-1021. See also R. M. Robinson, The approximation of irrational numbers by fractions with odd or even terms, Duke Math. J. vol. 7 (1940) pp. 354-359. 


$$
0<\frac{A}{B}-\xi<\frac{1}{\mu B^{2}}
$$

has infinitely many solutions, all but a finite number of which are convergents to $\xi$, if $\mu$ is a suitable number, depending on $\xi$. It is shown, in the theorem below, that $\mu$ may always be taken as one of the three numbers $1,4 / 3,2$. A similar result of course holds also for approximations from the left.

LEMma 1. Suppose $q_{n+1} \geqq 2$, so that $\lambda_{n}^{\prime}$ actually corresponds to a secondary convergent.

(a) If $\lambda_{n}^{\prime}>1$, then $\lambda_{n-1}>4 / 3$.

(b) If $\lambda_{n}^{\prime}>4 / 3$, then $\lambda_{n-1}>2$ or $\lambda_{n+1}>2$.

Proof. (a) From $\lambda_{n}^{\prime}>1$, we find $\beta_{n}<\alpha_{n}+2$. Hence

$$
\lambda_{n-1}=\beta_{n-1}+\frac{1}{\alpha_{n-1}}=\alpha_{n}+\frac{1}{\beta_{n}}>\alpha_{n}+\frac{1}{\alpha_{n}+2} .
$$

Since this expression increases with $\alpha_{n}$, which is at least 1 , we find that $\lambda_{n-1}>4 / 3$.

(b) Proof by contradiction. Suppose $\lambda_{n-1} \leqq 2$ and $\lambda_{n+1} \leqq 2$. From the first follows $\alpha_{n}<2$, and the second gives $\beta_{n+1}<2$, and hence

$$
\beta_{n}=q_{n+1}+\frac{1}{\beta_{n+1}}>2+\frac{1}{2} .
$$

Therefore

$$
\lambda_{n}^{\prime}=1+\frac{1}{\beta_{n}-1}-\frac{1}{\alpha_{n}+1}<1+\frac{2}{3}-\frac{1}{3}=\frac{4}{3} .
$$

LeMMA 2. Suppose $q_{n} \geqq 2$, so that $\lambda_{n}^{\prime \prime}$ actually corresponds to a secondary convergent.

(a) If $\lambda_{n}^{\prime \prime}>1$, then $\lambda_{n}>4 / 3$.

(b) If $\lambda_{n}^{\prime \prime}>4 / 3$, then $\lambda_{n}>2$ or $\lambda_{n-2}>2$.

Proof. (a) From $\lambda_{n}^{\prime \prime}>1$, we find $\alpha_{n}<\beta_{n}+2$, hence $\lambda_{n}>4 / 3$.

(b) Proof by contradiction. Suppose $\lambda_{n} \leqq 2$ and $\lambda_{n-2} \leqq 2$. From the first follows $\beta_{n}<2$, and the second gives $\alpha_{n-1}<2$ and hence $\alpha_{n}>5 / 2$. Therefore $\lambda_{n}^{\prime \prime}<4 / 3$.

Notice that both lemmas assert that if a secondary convergent gives a good approximation to $\xi$, then a convergent can be found (with a nearby subscript) giving an even better approximation to $\xi$ from the same side. 
ThEOREM. At least one of the three inequalities

$$
0<\frac{A}{B}-\xi<\frac{1}{B^{2}}, \quad 0<\frac{A}{B}-\xi<\frac{3}{4 B^{2}}, \quad 0<\frac{A}{B}-\xi<\frac{1}{2 B^{2}}
$$

has infinitely many solutions, of which all but a finite number are convergents to $\xi$.

Proof. The first inequality has infinitely many solutions. If it has infinitely many solutions which are not convergents, then they must be secondary convergents. Thus by the lemmas, corresponding convergents can be found satisfying the second inequality, which thus has infinitely many solutions. If it has infinitely many solutions which are not convergents, then they are again secondary convergents. Corresponding convergents can be found satisfying the third inequality, which thus has infinitely many solutions, all of which must be convergents.

4. The moduli of approximability. The degree of approximation possible for the irrational number $\xi$ is measured by the number

$$
M(\xi)=\lim \sup \lambda_{n},
$$

the modulus of approximability of the number $\xi$. In fact, the inequality

$$
\left|\frac{A}{B}-\xi\right|<\frac{1}{\mu B^{2}}
$$

has infinitely many solutions if $M(\xi)>\mu$, but not if $M(\xi)<\mu$. The function $M(\xi)$ has been studied by various authors. ${ }^{5}$ In a similar way, the degrees of approximation possible from the right and from the left are measured by

$$
M^{+}(\xi)=\lim \sup \lambda_{2 n+1}, \quad M^{-}(\xi)=\lim \sup \lambda_{2 n},
$$

which may be called the moduli of approximability from the right and left, respectively. Evidently

$$
M(\xi)=\max \left\{M^{+}(\xi), M^{-}(\xi)\right\} .
$$

Notice that

$$
M^{+}(-\xi)=M^{-}(\xi), \quad M^{-}(-\xi)=M^{+}(\xi),
$$

so that the roles of the two moduli are interchangeable.

- See J. F. Koksma, Diophantische Approximationen, Ergebnisse der Mathematik und ihrer Grenzgebiete, vol. 4, no. 4, Berlin, 1936, chap. 3, \$2. 
The numbers

$$
\xi_{k}=[k, 1, k, 1, k, 1, \cdots]
$$

will be of particular interest for our problem. It is clear that

$$
\begin{aligned}
M^{+}\left(\xi_{k}\right) & =[\cdots, 1, k, 1,[k], 1, k, 1, \cdots]=\rho_{k}, \\
M^{-}\left(\xi_{k}\right) & =[\cdots, k, 1, k,[1], k, 1, k, \cdots]=\sigma_{k} .
\end{aligned}
$$

Evaluating these continued fractions, we find that

$$
\rho_{k}=\left(k^{2}+4 k\right)^{1 / 2}, \quad \sigma_{k}=\left(k^{2}+4 k\right)^{1 / 2} / k .
$$

We noticed in $\S 1$ that it is sufficient to prove Segre's theorem for $0 \leqq \tau \leqq 1$. For these values of $\tau$, the error allowed on the right is less than or equal to the error allowed on the left. Now to prove that an inequality of the form

$$
-\frac{1}{\sigma B^{2}}<\frac{A}{B}-\xi<\frac{1}{\rho B^{2}},
$$

where $\rho \geqq \sigma$, has infinitely many solutions for each irrational number $\xi$, it is sufficient to consider those numbers for which $M^{+}(\xi) \geqq M^{-}(\xi)$. For if $M^{-}(\xi)>M^{+}(\xi)$, then $-\xi$ satisfies the condition imposed. Applying the inequality to $-\xi$, we see that we can satisfy

$$
-\frac{1}{\rho B^{2}}<\frac{A}{B}-\xi<\frac{1}{\sigma B^{2}}
$$

infinitely often. If $\rho=\sigma$, this is the required inequality. If $\rho>\sigma$, then $M^{+}(\xi) \geqq \sigma$ or $M^{-}(\xi) \geqq \rho$. Either of these conditions leads to $M^{-}(\xi)>\sigma$. Hence we can satisfy the inequality.

$$
-\frac{1}{\sigma B^{2}}<\frac{A}{B}-\xi<0
$$

infinitely often.

5. Classification of irrational numbers. In this section, we shall consider certain classes of irrational numbers, and show that together they include every irrational $\xi$ such that $M^{+}(\xi) \geqq M^{-}(\xi)$. The classes are defined by the following conditions, where $k$ denotes a positive integer.

(1 $\left.1_{k}\right) \quad q_{2 n} \rightarrow k, \quad q_{2 n+1} \rightarrow 1$, and hence $M^{+}(\xi)=\rho_{k}, M^{-}(\xi)=\sigma_{k}$.

(1 $\left.1_{\infty}\right) \quad q_{2 n} \rightarrow \infty, q_{2 n+1} \rightarrow 1$, and hence $M^{+}(\xi)=\infty, M^{-}(\xi)=1$.

$\left(2_{k}\right) \quad M^{+}(\xi)>\rho_{k}, M^{-}(\xi)>\sigma_{k}$. 


$$
\lim \sup q_{2 n}=k+1, \lim \inf q_{2 n}=k, q_{2 n+1} \rightarrow 1 \text {. }
$$

LEMmA. If $M^{+}(\xi) \geqq M^{-}(\xi)$, but we do not have $q_{2 n+1} \rightarrow 1$, then $\xi$ belongs to class $\left(2_{1}\right)$ or $\left(2_{2}\right)$.

Proof. Let $a=\lim \sup q_{2 n}, b=\lim \sup q_{2 n+1}$. By hypothesis, $b>1$. We have the following cases:

$$
\begin{aligned}
& b \geqq 3 . \quad M^{+}(\xi) \geqq M^{-}(\xi) \geqq 3 . \\
& b=2, a \geqq 4 . \quad M^{+}(\xi) \geqq 4, M^{-}(\xi) \geqq 2 . \\
& b=2, a \leqq 3 . \quad M^{+}(\xi) \geqq M^{-}(\xi) \geqq[4,[2], 4]=5 / 2 .
\end{aligned}
$$

Thus $\xi$ belongs to the classes $\left(2_{1}\right),\left(2_{2}\right),\left(2_{1}\right)$, respectively.

THEOREM. An irrational number $\xi$ with $M^{+}(\xi) \geqq M^{-}(\xi)$ belongs to at least one of the classes $\left(1_{k}\right),\left(1_{\infty}\right),\left(2_{k}\right),\left(3_{k}\right)$.

Proof. Because of the lemma, it will be sufficient to consider an irrational number $\xi$ with $q_{2 n+1} \rightarrow 1$, but not belonging to any class $\left(1_{k}\right)$, $\left(1_{\infty}\right)$, or $\left(3_{k}\right)$, and to show that it belongs to class $\left(2_{k}\right)$ for some $k$.

If $\lim \sup q_{2 n}=\infty$, we have $M^{+}(\xi)=\infty$. Since $\xi$ does not belong to the class $\left(1_{\infty}\right)$, we do not have $q_{2 n} \rightarrow \infty$, and hence $M^{-}(\xi)>1$. Thus $\xi$ belongs to class $\left(2_{k}\right)$ for any sufficiently large $k$.

It remains to consider the case when lim sup $q_{2 n}$ is finite. Since $\xi$ does not belong to any class $\left(1_{k}\right)$ or $\left(3_{k}\right)$, we must have

$$
\lim \sup q_{2 n}-\lim \inf q_{2 n} \geqq 2 \text {. }
$$

Hence there is an integer $k \geqq 2$, such that

$$
\lim \sup q_{2 n}=k+1, \quad \lim \inf q_{2 n} \leqq k-1 .
$$

From the first condition alone, it follows that

$$
M^{+}(\xi) \geqq[1,1,[k+1], 1,1]=k+2>\rho_{k} .
$$

Making use of the fact that infinitely many partial quotients do not exceed $k-1$, and that ultimately none exceed $k+1$, we also have

$$
M^{-}(\xi) \geqq[1, k+1,[1], k-1,1]=[k, 1, k,[1], k, 1, k]>\sigma_{k} \text {. }
$$

The inequalities here are clear, and the equality in the middle may be verified by actual expansion of the continued fractions. Thus $\xi$ belongs to $\operatorname{class}\left(2_{k}\right)$.

6. Segre's theorem. We shall put

$$
\rho_{\kappa}=\left(\kappa^{2}+4 \kappa\right)^{1 / 2}, \quad \sigma_{\kappa}=\left(\kappa^{2}+4 \kappa\right)^{1 / 2} / \kappa,
$$

even when $\kappa$ is not a positive integer. In particular, $\rho_{\infty}=\infty$ and $\sigma_{\infty}=1$. 
If we replace $\tau$ by $1 / \kappa$, Segre's inequality becomes

$$
-\frac{1}{\sigma_{\kappa} B^{2}}<\frac{A}{B}-\xi<\frac{1}{\rho_{k} B^{2}} .
$$

We are to show that this inequality has infinitely many solutions $A / B$ for each irrational number $\xi$ and each $\kappa$ such that $1 \leqq \kappa \leqq \infty$. As noted in $\$ 4$, for these values of $\kappa$ we need consider only values of $\xi$ such that $M^{+}(\xi) \geqq M^{-}(\xi)$. It is thus sufficient to prove the theorem for each of the classes defined in $\$ 5$.

For numbers of class $\left(1_{\infty}\right)$, it is clear that $\left(I_{\kappa}\right)$ is satisfied by infinitely many approximations from the right if $\kappa<\infty$, and by infinitely many approximations from the left if $\kappa=\infty$. For numbers of the other classes, the situation is made clearer by first formulating the following lemmas.

LEMMA 1. If for some $\kappa_{0}$ we have

$$
M^{+}(\xi)>\rho_{\kappa_{0}}, \quad M^{-}(\xi)>\sigma_{\kappa_{0}},
$$

then $\left(\mathrm{I}_{\kappa}\right)$ has infinitely many solutions for each $\kappa$.

Proof. Since $\rho_{\kappa}$ increases and $\sigma_{\kappa}$ decreases as $\kappa$ increases, we have $M^{+}(\xi)>\rho_{\kappa}$ for $\kappa \leqq \kappa_{0}$, and $M^{-}(\xi)>\sigma_{\kappa}$ for $\kappa \geqq \kappa_{0}$. Hence $\left(\mathrm{I}_{\kappa}\right)$ is satisfied by infinitely many approximations from the right if $\kappa \leqq \kappa_{0}$, and by infinitely many approximations from the left if $\kappa \geqq \kappa_{0}$.

LEMMA 2. If for some $\kappa_{0}$ we have

$$
M^{+}(\xi)=\rho_{\kappa_{0}}, \quad M^{-}(\xi)=\sigma_{\kappa_{0}},
$$

then $\left(\mathrm{I}_{\kappa}\right)$ has infinitely many solutions for each $\kappa$, except possibly for $\kappa=\kappa_{0}$.

Proof. $M^{+}(\xi)>\rho_{\kappa}$ for $\kappa<\kappa_{0}$, and $M^{-}(\xi)>\sigma_{\kappa}$ for $\kappa>\kappa_{0}$.

For numbers of class $\left(2_{k}\right)$, the hypothesis of Lemma 1 is satisfied with $\kappa_{0}=k$. Hence the desired conclusion follows. For numbers of class $\left(1_{k}\right)$, the hypothesis of Lemma 2 is satisfied with $\kappa_{0}=k$. Hence the conclusion follows for $\kappa \neq k$. Now for numbers of class $\left(1_{k}\right)$, we have

$$
\lambda_{2 n+1} \rightarrow \rho_{k}, \quad \lambda_{2 n} \rightarrow \sigma_{k},
$$

one of the limits being approached from above and the other from below. Hence for numbers of class $\left(1_{k}\right)$, the inequality $\left(\mathrm{I}_{k}\right)$ can also be satisfied.

It remains to prove $\left(\mathrm{I}_{\kappa}\right)$ for numbers of class $\left(3_{k}\right)$. We first notice that for numbers of this class 


$$
\rho_{k} \leqq M^{+}(\xi) \leqq \rho_{k+1}, \quad \sigma_{k+1} \leqq M^{-}(\xi) \leqq \sigma_{k} .
$$

The upper bounds here are sharp, but the lower bounds are not. It is easily seen that the sharp lower bounds are given by

$$
\begin{aligned}
& M^{+}(\xi) \geqq[\cdots, k, 1, k, 1,[k+1], 1, k, 1, k, \cdots]=\rho_{k}^{\prime}, \\
& M^{-}(\xi) \geqq[\cdots, k+1,1, k+1,[1], k, 1, k+1, \cdots]=\sigma_{k+1}^{\prime} .
\end{aligned}
$$

It is clear that

$$
\rho_{k}^{\prime}=1+\rho_{k}=1+\left(k^{2}+4 k\right)^{1 / 2} .
$$

Replacing $k$ by $k-1$ in the other expression and expanding, we find that for $k \geqq 2$,

$$
\sigma_{k}^{\prime}=\frac{k(k+1)+(3 k-1)\left(k^{2}+4 k\right)^{1 / 2}}{2 k(2 k-1)} .
$$

We shall show below that

$$
\rho_{k}^{\prime}>\rho_{k+0.8}, \quad \sigma_{k}^{\prime}>\sigma_{k-0.5} .
$$

From these inequalities, it follows that

$$
M^{+}(\xi) \geqq \rho_{k}^{\prime}>\rho_{k+0.8}, \quad M^{-}(\xi) \geqq \sigma_{k+1}^{\prime}>\sigma_{k+0.5},
$$

for any number $\xi$ of class $\left(3_{k}\right)$. Hence the hypothesis of Lemma 1 is satisfied for numbers of class $\left(3_{k}\right)$ with a suitable $\kappa_{0}$, and indeed with any $\kappa_{0}$ such that

$$
k+0.5 \leqq \kappa_{0} \leqq k+0.8 .
$$

The proof of the theorem will therefore be complete.

PROOF THAT $\rho_{k}^{\prime}>\rho_{k+0.8}$. The inequality to be proved is

$$
1+\left(k^{2}+4 k\right)^{1 / 2}>\left\{(k+0.8)^{2}+4(k+0.8)\right\}^{1 / 2} .
$$

If we square and simplify, this reduces to

$$
50\left(k^{2}+4 k\right)^{1 / 2}>40 k+71 .
$$

Squaring again, we have

$$
900 k^{2}+4320 k>5041,
$$

which is true, since the left side is at least 5220 .

PROOF THAT $\sigma_{k}^{\prime}>\sigma_{k-0.5}$. If we multiply through by $2 k(2 k-1)$, the required inequality takes the form

$$
k(k+1)+(3 k-1)\left(k^{2}+4 k\right)^{1 / 2}>2 k\left\{(2 k-1)^{2}+8(2 k-1)\right\}^{1 / 2} .
$$


If we square and simplify, this reduces to

$$
(3 k-1)(k+1)\left(k^{2}+4 k\right)^{1 / 2}>(3 k-1) k(k+3)-2,
$$

which is certainly true if

$$
(k+1)\left(k^{2}+4 k\right)^{1 / 2}>k(k+3) .
$$

Squaring this, we obtain the inequality

$$
k^{4}+6 k^{3}+9 k^{2}+4 k>k^{4}+6 k^{3}+9 k^{2},
$$

which is true.

7. Some stronger results. The inequality

$$
-\frac{1}{\sigma_{k} B^{2}}<\frac{A}{B}-\xi<\frac{1}{\rho_{k} B^{2}},
$$

where $k$ is a positive integer, is a special case of Segre's inequality. Now for numbers of class $\left(1_{k}\right)$, we have

$$
M^{+}(\xi)=\rho_{k}, \quad M^{-}(\xi)=\sigma_{k} .
$$

It follows, as noted by Segre, that we cannot increase both of the numbers $\rho_{k}$ and $\sigma_{k}$. Actually, for numbers of the class $\left(1_{k}\right)$, we have

$$
\lambda_{2 n+1} \rightarrow \rho_{k}, \quad \lambda_{2 n} \rightarrow \sigma_{k},
$$

one of the limits being approached from above and the other from below; which one is approached from which side depends on the beginning of the continued fraction expansion. Hence we cannot increase either of the numbers $\rho_{k}$ or $\sigma_{k}$, leaving the other one fixed.

Nevertheless, there are infinitely many solutions to the inequality

$$
-\frac{1}{\left(\sigma_{k}-\epsilon\right) B^{2}}<\frac{A}{B}-\xi<\frac{1}{\rho_{k}^{\prime} B^{2}},
$$

for any $\epsilon>0$, although $\rho_{k}^{\prime}=\rho_{k}+1$. In a similar way, if $k \geqq 2$ the inequality

$$
-\frac{1}{\sigma_{k}^{\prime} B^{2}}<\frac{A}{B}-\xi<\frac{1}{\left(\rho_{k}-\epsilon\right) B^{2}}
$$

has infinitely many solutions for each $\epsilon>0$, although $\sigma_{k}^{\prime}>\sigma_{k}$. We shall confine our discussion to the inequality $\left(\mathrm{I}_{k}^{\prime}\right)$, since the proof of $\left(\mathrm{I}_{k}^{\prime \prime}\right)$ is entirely similar.

To prove the inequality $\left(\mathrm{I}_{k}^{\prime}\right)$, we need consider only numbers $\xi$ for which $M^{+}(\xi) \geqq M^{-}(\xi)$, as shown in $\S 4$. Now $\left(\mathrm{I}_{k}^{\prime}\right)$ is satisfied by approximations from the right for numbers of classes $\left(1_{j}\right),\left(2_{j}\right)$, and $\left(3_{j}\right)$, 
if $j>k$, since $\rho_{j} \geqq \rho_{k+1}>\rho_{k}^{\prime}$. It is satisfied by approximations from the left for numbers of the classes $\left(1_{j}\right)$ and $\left(2_{j}\right)$ if $j \leqq k$, since $\sigma_{j}>\sigma_{k}-\epsilon$, and for numbers of class $\left(3_{j}\right)$ if $j<k$, since then $\sigma_{j+1}>\sigma_{k}-\epsilon$.

It remains only to consider the numbers of class $\left(3_{k}\right)$. For these numbers we have $M^{+}(\xi) \geqq \rho_{k}^{\prime}$, and indeed $\lambda_{2 n+1}>\rho_{k}^{\prime}$ has infinitely many solutions, since it is true whenever $n$ is sufficiently large and $q_{2 n+2}=k+1$. Thus in this case, $\left(\mathrm{I}_{k}^{\prime}\right)$ can be satisfied by approximations from the right.

It is easily seen that there are numbers of class $\left(3_{k}\right)$ for which $M^{-}(\xi)<\sigma_{k}$ and $M^{+}(\xi)$ is arbitrarily near to $\rho_{k}^{\prime}$. It follows that we cannot replace $\rho_{k}^{\prime}$ by any larger constant on the right side of the inequality $\left(\mathrm{I}_{k}^{\prime}\right)$.

A particularly interesting case of the theorem just proved is obtained by putting $k=1$ : For any $\epsilon>0$, every irrational number $\xi$ has infinitely many approximations $A / B$ such that

$$
-\frac{1}{\left(5^{1 / 2}-\epsilon\right) B^{2}}<\frac{A}{B}-\xi<\frac{1}{\left(5^{1 / 2}+1\right) B^{2}} .
$$

The coefficient of $B^{2}$ in the denominator on the right cannot be replaced by any larger constant.

UNIVERSITY OF CALIFornia AND

Princeton University 